\title{
Common fixed point theorems concerning F-contraction in b-metric-like spaces
}

\author{
Chunfang Chen, Huiling Xue, Chuanxi Zhu* \\ Department of Mathematics, Nanchang University, Nanchang, 330031, jiangxi, P. R. China. \\ Communicated by F. Vetro
}

\begin{abstract}
In this work, some new types of F-contraction are introduced in partially ordered b-metric-like spaces and some common fixed point theorems concerning F-contraction are investigated. Moreover, we give an example to illustrate the availability of the obtained results. (C)2017 All rights reserved.
\end{abstract}

Keywords: b-metric-like spaces, common fixed point, fixed point, F-contraction.

2010 MSC: 47H10, 54H25.

\section{Introduction and preliminaries}

In this paper, $\mathbb{N}, \mathbb{N}^{+}, \mathbb{R}, \mathbb{R}_{0}^{+}$, and $\mathbb{R}^{+}$are used to denote the set of all nonnegative integer numbers, the set of all positive integer numbers, the set of all real numbers, the set of all nonnegative real numbers, and the set of all positive real numbers, respectively.

After the appearance of the Banach contractive mapping principle in metric spaces, many authors investigated various generalizations of metric spaces (such as partial metric spaces [9] and b-metric spaces [6]) and researched on the Banach contractive mapping principle in generalized metric spaces. In [2], as a new generalization of metric spaces, b-metric-like spaces was introduced as follows.

Definition 1.1 ([2]). A b-metric-like on a nonempty set $X$ is a function $\sigma: X \times X \rightarrow \mathbb{R}_{0}^{+}$such that the following three conditions hold true:

$\left(\sigma_{1}\right)$ if $\sigma(x, y)=0$ then $x=y$;

$\left(\sigma_{2}\right) \sigma(x, y)=\sigma(y, x)$;

$\left(\sigma_{3}\right) \sigma(x, z) \leqslant s(\sigma(x, y)+\sigma(y, z))$

for all $x, y, z \in X$ and a constant $s \geqslant 1$. The pair $(X, \sigma)$ is then called a b-metric-like space.

\footnotetext{
*Corresponding author $\mathrm{Zhu}$ )

Email addresses: ccfygd@sina.com (Chunfang Chen), 1528548445@qq. com (Huiling Xue), chuanxizhu@126. com (Chuanxi
}

doi:10.22436/jnsa.010.06.22 
Each b-metric-like $\sigma$ on $X$ generalizes a topology $\tau_{\sigma}$ on $X$ whose base is the family of open $\sigma$-balls $B_{\sigma}(x, \varepsilon)=\{y \in X:|\sigma(x, y)-\sigma(x, x)|<\varepsilon\}$ for all $x \in X$ and $\varepsilon>0$.

Example 1.2 ([2]). Let $X=\mathbb{R}_{0}^{+}$. The function $\sigma: X \times X \rightarrow \mathbb{R}_{0}^{+}$is defined by $\sigma(x, y)=(x+y)^{2}$. Then $(X, \sigma)$ is a $b$-metric-like space with the constant $s=2$.

The following concepts in b-metric-like spaces can be found in [2].

In the $b$-metric-like space $(X, \sigma)$, a sequence $\left\{x_{n}\right\}$ converges to a point $x \in X$ if and only if $\sigma(x, x)=$ $\lim _{n \rightarrow+\infty} \sigma\left(x, x_{n}\right)$.

In the b-metric-like space $(X, \sigma)$, if there exists $\lim _{n, m \rightarrow+\infty} \sigma\left(x_{m}, x_{n}\right)$ and it is finite, then the sequence $\left\{x_{n}\right\}$ is called to be a Cauchy sequence.

A b-metric-like space $(X, \sigma)$ is called to be complete if every Cauchy sequence $\left\{x_{n}\right\}$ in $X$ converges with respect to $\tau_{\sigma}$ to a point $x \in X$ such that $\lim _{n \rightarrow+\infty} \sigma\left(x, x_{n}\right)=\sigma(x, x)=\lim _{n \rightarrow+\infty} \sigma\left(x_{m}, x_{n}\right)$.

On the other hand, the concept of F-contraction was introduced by Wardowski in [12], at the same time, Wardowski investigated a fixed point result, which is a generalization of the Banach contraction principle.

We use $\mathbb{F}$ to denote the set of all functions satisfying the following conditions (F1)-(F3).

(F1) $F$ is strictly increasing, that is, for all $\alpha, \beta \in \mathbb{R}^{+}$such that $\alpha<\beta, F(\alpha)<F(\beta)$;

(F2) for any sequence $\left\{\alpha_{n}\right\}$ of positive real numbers, the following holds:

$$
\lim _{n \rightarrow+\infty} \alpha_{n}=0 \text { if and only if } \lim _{n \rightarrow+\infty} F\left(\alpha_{n}\right)=-\infty \text {; }
$$

(F3) there exists $k \in(0,1)$ such that $\lim _{\alpha \rightarrow 0^{+}} \alpha^{\mathrm{k}} \mathrm{F}(\alpha)=0$.

Wardowski defined the F-contraction as follows.

Definition 1.3 ([12]). Let $(X, d)$ be a metric space. A mapping $T: X \rightarrow X$ is said to be an F-contraction if there exist $F \in \mathbb{F}$ and $\tau>0$ such that

$$
d(T x, T y)>0 \Rightarrow \tau+F(d(T x, T y)) \leqslant F(d(x, y))
$$

for all $x, y \in X$.

After that, F-contraction was generalized and a lot of fixed point theorems concerning F-contraction were obtained [3-5, 7, 8, 11]. Recently, Wardowski and Dung [13] introduced the notion of an F-weak contraction and investigated a fixed point theorem for such contraction in metric spaces.

Definition $1.4([13])$. Let $(X, d)$ be a metric space, and $T: X \rightarrow X$ be a mapping. If there exist $\tau>0$ and $\mathrm{F} \in \mathbb{F}$ such that

$$
d(T x, T y)>0 \Rightarrow \tau+F(d(T x, T y)) \leqslant F\left(\max \left\{d(x, y), d(x, T x), d(y, T y), \frac{d(x, T y)+d(y, T x)}{2}\right\}\right)
$$

for all $x, y \in X$, then $T$ is said to be an F-weak contraction.

Let $(X, \preceq)$ be a partial ordered set and $x, y \in X$. If $x \preceq y$ or $y \preceq x$ holds, then $x$ and $y$ are called to be comparable, we will write $x \asymp y$, which is consistent with [8].

Definition 1.5 ([8]). Let $(X, \preceq)$ be a partial ordered set and $g$ be a self-mapping on $X$. The set $\mathbb{A}$ of $X$ is said to be well-ordered if $x \asymp y$ for all $x, y \in \mathbb{A}$. If $g x \asymp g y$ for all $x, y \in \mathbb{A}$, then $\mathbb{A}$ is called to be g-well-ordered.

Malhotra et al. [8] introduced ordered F-g-weak contraction in metric spaces as follows. 
Definition $1.6([8])$. Let $(X, \sigma, \preceq)$ be a partially ordered metric-like space, and $f$ and $g$ be self-mappings on $X$. If there exist $\tau>0$ and $F \in \mathbb{F}$ such that

$$
\sigma(f x, f y)>0 \Rightarrow \tau+F(\sigma(f x, f y)) \leqslant F(\max \{\sigma(g x, g y), \sigma(g x, f x), \sigma(g y, f y)\})
$$

for all $x, y \in X$ with $g x \asymp g y$, then the mapping $f$ is called to be an ordered F-g-weak contraction.

Recently, Piri and Kumam [10] investigated some fixed point theorems concerning F-contraction in complete metric spaces by replacing the condition (F3) with the condition: $\mathrm{F}$ is continuous on $\mathbb{R}^{+}$.

In this work, inspired by Definitions 1.4 and 1.6, by replacing the condition (F3) with the condition: $\mathrm{F}$ is continuous on $\mathbb{R}^{+}$, we introduce some new types of generalized ordered F-g-weak contraction in b-metric-like spaces and investigate some fixed point theorems for these generalized ordered F-g-weak contractions.

\section{Main results}

In this section, we begin with the following definitions which will be used in the sequel.

Definition 2.1. Let $\widetilde{F}$ be the family of all functions $F: \mathbb{R}^{+} \rightarrow \mathbb{R}$ satisfying the following conditions:

(F1) $F$ is strictly increasing, that is, $F(\alpha)<F(\beta)$ for all $\alpha, \beta \in \mathbb{R}^{+}$such that $\alpha<\beta$;

(F2) for every sequence $\left\{\alpha_{n}\right\}$ of positive real numbers, the following holds:

$$
\lim _{n \rightarrow+\infty} \alpha_{n}=0 \text { if and only if } \lim _{n \rightarrow+\infty} F\left(\alpha_{n}\right)=-\infty \text {; }
$$

(F3) $\mathrm{F}$ is continuous.

Definition 2.2. Let $(X, \sigma, \preceq)$ be a partially ordered b-metric-like space, where $\preceq$ is a partial order on $X$ and $\sigma$ is a b-metric-like on $X$, and let $f$ and $g$ be self-mappings on $X$. Assume that there exist $\tau>0$ and $\mathrm{F} \in \widetilde{\mathbb{F}}$ such that

$$
\sigma(f x, f y)>0 \Rightarrow \tau+F\left(s^{2} \sigma(f x, f y)\right) \leqslant F\left(\max \left\{\sigma(g x, g y), \sigma(g x, f x), \sigma(g y, f y), \frac{\sigma(g x, f y)+\sigma(g y, f x)}{4 s}\right\}\right)
$$

for all $x, y \in X$ with $g x \asymp g y$. Then the mapping $f$ is called to be a generalized ordered F-g-weak contraction of type (A).

Definition 2.3 ([1]). Let $X$ be a nonempty set, $f$ and $g$ be self-mappings on $X$, and $C(f, g)=\{x \in X: f x=$ $g x\}$. The pair $f$ and $g$ are called to be weakly compatible if $f g x=g f x$ for all $x \in C(f, g)$. If $w=f x=g x$ for some $x \in X$, then $x$ is called to be a coincidence of $f$ and $g$, and $w$ is called to be a point of coincidence of $f$ and $g$.

In the proof of our main results, we will need the following lemma.

Lemma 2.4. Let $(X, \sigma, \preceq)$ be a partially ordered b-metric-like space, and $f$ and $g$ be self-mappings on $X$ such that $\mathrm{f}$ is a generalized ordered F-g-weak contraction of type (A). If $v \in X$ is a point of coincidence of $\mathrm{f}$ and $\mathrm{g}$, then $\sigma(v, v)=0$.

Proof. Suppose that $v \in X$ is a point of coincidence of $f$ and $g$, then there exists $u \in X$ such that $f u=g u=$ $v$. Now, we prove $\sigma(v, v)=0$. Suppose $\sigma(v, v)>0$, using the fact that $f$ is a generalized ordered F-g-weak contraction of type (A), we have

$$
\begin{aligned}
\tau+\mathrm{F}\left(\mathrm{s}^{2} \sigma(v, v)\right)=\tau+\mathrm{F}\left(\mathrm{s}^{2} \sigma(\mathrm{fu}, \mathrm{fu})\right) & \leqslant \mathrm{F}\left(\max \left\{\sigma(\mathrm{gu}, \mathrm{gu}), \sigma(\mathrm{gu}, \mathrm{fu}), \sigma(\mathrm{gu}, \mathrm{fu}), \frac{\sigma(\mathrm{gu}, \mathrm{fu})+\sigma(\mathrm{gu}, \mathrm{fu})}{4 \mathrm{~s}}\right\}\right) \\
& =\mathrm{F}\left(\max \left\{\sigma(v, v), \sigma(v, v), \sigma(v, v), \frac{\sigma(v, v)+\sigma(v, v)}{4 s}\right\}\right)=\mathrm{F}(\sigma(v, v)),
\end{aligned}
$$

which together with $\mathrm{F}(1)$ yields $s^{2} \sigma(v, v)<\sigma(v, v)$. This is a contradiction with $s \geqslant 1$. Therefore, we get $\sigma(v, v)=0$. 
Now, we start to prove our main results.

Theorem 2.5. Let $(\mathrm{X}, \sigma, \preceq)$ be a partially ordered b-metric-like space, $\mathrm{f}$ and $\mathrm{g}$ be self-mappings on $\mathrm{X}$ such that $\mathrm{f}$ is a generalized ordered F-g-weak contraction of type $(A)$ with $\mathrm{f}(\mathrm{X}) \subseteq \mathrm{g}(\mathrm{X})$, and $\mathrm{g}(\mathrm{X})$ is complete. If the following conditions hold:

(i) $\mathrm{fx} \preceq$ fy for $x, y \in X$ such that $g x \preceq g y$;

(ii) there exists $\mathrm{x}_{0} \in \mathrm{X}$ such that $\mathrm{gx}_{0} \asymp \mathrm{fx}_{0}$;

(iii) if $\left\{x_{n}\right\}$ is a sequence in $(X, \sigma)$ which converges to $x \in X$ and $\left\{x_{n}: n \in \mathbb{N}\right\}$ is well-ordered, then $x_{n} \asymp x$ for sufficiently large $n$,

then $\mathrm{f}$ and $\mathrm{g}$ have a point of coincidence $v \in \mathrm{X}$ and $\sigma(v, v)=0$. Furthermore, if the set of coincidence points of $\mathrm{f}$ and $\mathrm{g}$ is $\mathrm{g}$-well-ordered, then $\mathrm{f}$ and $\mathrm{g}$ have a unique point of coincidence. In addition, if $\mathrm{f}$ and $\mathrm{g}$ are weakly compatible, then $f$ and $g$ have a unique common fixed point.

Proof. For $x_{0} \in X$, taking $f(X) \subseteq g(X)$ into account, we can construct a sequence $\left\{y_{n}\right\}$ by $y_{n}=g x_{n}=f x_{n-1}$ for $n \in \mathbb{N}^{+}$. Since $g x_{0} \asymp f x_{0}=g x_{1}$, then $g x_{0} \preceq f x_{0}=g x_{1}$ or $f x_{0}=g x_{1} \preceq g x_{0}$. If $g x_{0} \preceq f x_{0}=g x_{1}$, from (i), we have $f x_{0} \preceq f x_{1}$, that is $g x_{1} \preceq f x_{1}=g x_{2}$, hence $f x_{1} \preceq f x_{2}$ by (i). On repeating this process, we get

$$
y_{n}=g x_{n} \preceq g x_{n+1}=y_{n+1}
$$

for all $n \in \mathbb{N}$. Similarly, if $f x_{0}=g x_{1} \preceq g x_{0}$, we can get $y_{n+1} \preceq y_{n}$ for all $n \in \mathbb{N}$. From the above, we obtain that $\left\{y_{n}: n \in \mathbb{N}\right\}$ is well-ordered. Now, we claim that $f$ and $g$ have a point of coincidence. If $\sigma\left(y_{n}, y_{n+1}\right)=0$ for some $n_{0} \in \mathbb{N}$, i.e., $\sigma\left(y_{n_{0}}, y_{n_{0}+1}\right)=0$, that is $y_{n_{0}}=y_{n_{0}+1}$, it implies $g x_{n_{0}+1}=f x_{n_{0}-1}$, which together with $g x_{n_{0}}=f x_{n_{0}-1}$ and $g x_{n_{0}+1}=f x_{n_{0}}$ yields $g x_{n_{0}}=f x_{n_{0}}$. Therefore, $x_{n_{0}}$ is a coincidence point of $f$ and $g$ and $g x_{n_{0}}=f x_{n_{0}}$ is a point of coincidence of $f$ and $g$. Hence, we assume $\sigma\left(y_{n}, y_{n+1}\right)>0$ for all $n \in \mathbb{N}$. Applying (2.1) and (2.2), we obtain

$$
\begin{aligned}
\tau+F\left(s^{2} \sigma\left(y_{n}, y_{n+1}\right)\right) \\
=\tau+F\left(s^{2} \sigma\left(f x_{n-1}, f x_{n}\right)\right) \\
\leqslant F\left(\max \left\{\sigma\left(g x_{n-1}, g x_{n}\right), \sigma\left(g x_{n-1}, f x_{n-1}\right), \sigma\left(g x_{n}, f x_{n}\right), \frac{\sigma\left(g x_{n-1}, f x_{n}\right)+\sigma\left(g x_{n}, f x_{n-1}\right)}{4 s}\right\}\right) \\
=F\left(\max \left\{\sigma\left(y_{n-1}, y_{n}\right), \sigma\left(y_{n-1}, y_{n}\right), \sigma\left(y_{n}, y_{n+1}\right), \frac{\sigma\left(y_{n-1}, y_{n+1}\right)+\sigma\left(y_{n}, y_{n}\right)}{4 s}\right\}\right) \\
\leqslant F\left(\max \left\{\sigma\left(y_{n-1}, y_{n}\right), \sigma\left(y_{n-1}, y_{n}\right), \sigma\left(y_{n}, y_{n+1}\right), \frac{s \sigma\left(y_{n-1}, y_{n}\right)+3 s \sigma\left(y_{n}, y_{n+1}\right)}{4 s}\right\}\right) \\
\leqslant F\left(\operatorname { m a x } \left\{\sigma\left(y_{n-1}, y_{n}\right), \sigma\left(y_{n-1}, y_{n}\right), \sigma\left(y_{n}, y_{n+1}\right),\right.\right. \\
\left.\left.\quad \quad \frac{\max \left\{\sigma\left(y_{n-1}, y_{n}\right), \sigma\left(y_{n}, y_{n+1}\right)\right\}+3 s \max \left\{\sigma\left(y_{n-1}, y_{n}\right), \sigma\left(y_{n}, y_{n+1}\right)\right\}}{4 s}\right\}\right) .
\end{aligned}
$$

If there exists $\mathrm{n}_{0} \in \mathbb{N}^{+}$such that $\max \left\{\sigma\left(\mathrm{y}_{n_{0}-1}, \mathrm{y}_{n_{0}}\right), \sigma\left(\mathrm{y}_{n_{0}}, y_{n_{0}+1}\right)\right\}=\sigma\left(y_{n_{0}}, y_{n_{0}+1}\right)$, by (2.3), we get $\tau+F\left(s^{2} \sigma\left(y_{n_{0}}, y_{n_{0}+1}\right)\right) \leqslant F\left(\sigma\left(y_{n_{0}}, y_{n_{0}+1}\right)\right)$, which shows $s^{2} \sigma\left(y_{n_{0}}, y_{n_{0}+1}\right)<\sigma\left(y_{n_{0}}, y_{n_{0}+1}\right)$, this is a contradiction since $s \geqslant 1$, thus we get $\max \left\{\sigma\left(y_{n-1}, y_{n}\right), \sigma\left(y_{n}, y_{n+1}\right)\right\}=\sigma\left(y_{n-1}, y_{n}\right)$ for all $n \in \mathbb{N}^{+}$. From (2.3), we have $\tau+F\left(\sigma\left(y_{n}, y_{n+1}\right)\right) \leqslant \tau+F\left(s^{2} \sigma\left(y_{n}, y_{n+1}\right)\right) \leqslant F\left(\sigma\left(y_{n-1}, y_{n}\right)\right)$, thus, for all $n \in \mathbb{N}^{+}$, we have

$$
F\left(\sigma\left(y_{n}, y_{n+1}\right)\right) \leqslant F\left(\sigma\left(y_{n-1}, y_{n}\right)\right)-\tau \text {. }
$$

Repeating this process, we obtain

$$
F\left(\sigma\left(y_{n}, y_{n+1}\right)\right) \leqslant F\left(\sigma\left(y_{0}, y_{1}\right)\right)-n \tau .
$$

Taking $n \rightarrow+\infty$, (2.4) shows $\lim _{n \rightarrow+\infty} F\left(\sigma\left(y_{n}, y_{n+1}\right)\right)=-\infty$, hence,

$$
\lim _{n \rightarrow+\infty} \sigma\left(y_{n}, y_{n+1}\right)=0 .
$$


Now, we prove

$$
\lim _{m, n \rightarrow+\infty} \sigma\left(y_{m}, y_{n}\right)=0
$$

In fact, if $\lim _{m, n \rightarrow+\infty} \sigma\left(y_{m}, y_{n}\right) \neq 0$, there exist $\varepsilon>0$ and sequences $\{p(n)\}$ and $\{q(n)\}$ of natural numbers such that for all $n \in \mathbb{N}$, the following inequalities hold.

$$
p(n)>q(n)>n, \quad \sigma\left(y_{p(n)}, y_{q(n)}\right) \geqslant \varepsilon, \quad \sigma\left(y_{p}(n)-1, y_{q(n)}\right)<\varepsilon .
$$

Applying the triangle inequality and (2.7), we get

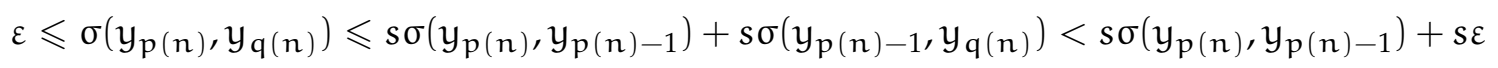

for all $n \in \mathbb{N}$. Taking (2.5) into account in the above inequalities, we obtain that the sequence $\left\{\sigma\left(y_{\mathfrak{p}(n)}, y_{q}(n)\right)\right\}$ is bounded, so, it has inferior limit, and we let

$$
\liminf _{n \rightarrow+\infty} \sigma\left(y_{p}(n), y_{q(n)}\right)=\underline{A} .
$$

From the triangle inequality, we have

$$
\sigma\left(y_{p(n)+1}, y_{q(n)+1}\right) \leqslant s \sigma\left(y_{p(n)+1}, y_{p(n)}\right)+s^{2} \sigma\left(y_{p(n)}, y_{q(n)}\right)+s^{2} \sigma\left(y_{q(n)}, y_{q(n)+1}\right),
$$

which together with (2.5) shows that the sequence $\left\{\sigma\left(y_{p(n)+1}, y_{q(n)+1}\right)\right\}$ is bounded, hence the sequence $\left\{\sigma\left(y_{\mathfrak{p}(n)+1}, y_{\mathfrak{q}(n)+1}\right)\right\}$ has inferior limit. Assume

$$
\liminf _{n \rightarrow+\infty} \sigma\left(y_{p}(n)+1, y_{q}(n)+1\right)=\underline{B} .
$$

Due to the triangle inequality, we get

$$
\sigma\left(y_{p(n)}, y_{q(n)}\right) \leqslant s \sigma\left(y_{p(n)}, y_{p(n)+1}\right)+s^{2} \sigma\left(y_{p(n)+1}, y_{q(n)+1}\right)+s^{2} \sigma\left(y_{q(n)+1}, y_{q(n)}\right),
$$

Letting $n \rightarrow+\infty$ in the above inequality, by (2.5), we have

$$
\underline{A} \leqslant s^{2} \underline{B} .
$$

Combining (2.7), (2.8), and (2.9), we obtain $\underline{B} \geqslant \frac{A}{s^{2}} \geqslant \frac{\varepsilon}{s^{2}}$, hence, we can assume that there exists $N_{1} \in \mathbb{N}$ such that $\sigma\left(y_{p(n)+1}, y_{q(n)+1}\right)>\frac{\varepsilon}{2 s^{2}}>0$ for all $n>N_{1}$, therefore, by (2.1), we get

$$
\begin{aligned}
\tau+ & F\left(s^{2} \sigma\left(y_{p(n)+1}, y_{q}(n)+1\right)\right) \\
= & \tau+F\left(s^{2} \sigma\left(f x_{p(n)}, f x_{q(n)}\right)\right) \\
\leqslant & F\left(\max _{\{} \sigma\left(g x_{p(n)}, g x_{q(n)}\right), \sigma\left(g x_{p(n)}, f x_{p(n)}\right), \sigma\left(g x_{q(n)}, f x_{q(n)}\right),\right. \\
& \left.\left.\quad \frac{\sigma\left(g x_{p(n)}, f x_{q(n)}\right)+\sigma\left(g x_{q(n)}, f x_{p(n)}\right)}{4 s}\right\}\right) \\
= & F\left(\operatorname { m a x } \left\{\sigma\left(y_{p(n)}, y_{q(n)}\right), \sigma\left(y_{p}(n), y_{p(n)+1}\right), \sigma\left(y_{q(n)}, y_{q(n)+1}\right),\right.\right. \\
& \left.\left.\frac{\sigma\left(y_{p(n)}, y_{q(n)+1}\right)+\sigma\left(y_{q(n)}, y_{p(n)+1}\right)}{4 s}\right\}\right) \\
\leqslant & F\left(\operatorname { m a x } \left\{\sigma\left(y_{p(n)}, y_{q}(n)\right), \sigma\left(y_{p}(n), y_{p(n)+1}\right), \sigma\left(y_{q(n)}, y_{q(n)+1}\right),\right.\right. \\
& \left.\left.\quad \frac{s \sigma\left(y_{p(n)}, y_{q(n)}\right)+s \sigma\left(y_{q(n)}, y_{q(n)+1}\right)+s \sigma\left(y_{q(n)}, y_{p(n)}\right)+s \sigma\left(y_{p}(n), y_{p}(n)+1\right)}{4 s}\right\}\right) .
\end{aligned}
$$


Since $F$ is continuous, taking $n \rightarrow+\infty$ in (2.10), we obtain $\tau+F\left(s^{2} \underline{B}\right) \leqslant F(\underline{A})$, which means $s^{2} \underline{B}<\underline{A}$, this is a contradiction with (2.9), thus (2.6) holds. Therefore the sequence $\left\{y_{n}\right\}=\left\{g x_{n}\right\}$ is a Cauchy sequence in $g(X)$. Since $g(X)$ is complete, there exist $u, v \in X$ such that $v=g u$ and

$$
\lim _{n \rightarrow+\infty} \sigma\left(y_{n}, v\right)=\lim _{n \rightarrow+\infty} \sigma\left(g x_{n}, g u\right)=\lim _{m, n \rightarrow+\infty} \sigma\left(y_{n}, y_{m}\right)=\sigma(v, v)=0 .
$$

Now, we claim $\sigma(f u, v)=0$. In fact, if $\sigma(f u, v)>0$, applying the triangle inequality, we get

$$
\sigma\left(y_{n}, f u\right) \leqslant s \sigma\left(y_{n}, v\right)+s \sigma(v, f u)
$$

it shows that $\sigma\left(y_{n}, f u\right)$ is bounded due to (2.11), hence the sequence $\left\{\sigma\left(y_{n}, f u\right)\right\}$ has inferior limit, we assume

$$
\liminf _{n \rightarrow+\infty} \sigma\left(y_{n}, f u\right)=\underline{C} .
$$

By the triangle inequality, we have

$$
\sigma(v, f u) \leqslant s \sigma\left(y_{n}, v\right)+s \sigma\left(y_{n}, f u\right),
$$

taking $\mathrm{n} \rightarrow+\infty$ in the above inequality, by (2.11), we obtain $\liminf _{n \rightarrow+\infty} \sigma\left(y_{n}, f u\right)=\underline{C} \geqslant \frac{\sigma(v, f u)}{s}$, thus, we can assume that there exists $\mathrm{N}_{2} \in \mathbb{N}$ such that $\sigma\left(\mathrm{y}_{n}, \mathrm{fu}\right)>\frac{\sigma(v, \mathrm{fu})}{2 \mathrm{~s}}$ for $\mathrm{n}>\mathrm{N}_{2}$. Since $\sigma(v, f u)>0$, then $\sigma\left(y_{n}, f u\right)>0$ for all $n>N_{2}$. Applying (2.1) and condition (iii), we have

$$
\begin{aligned}
\tau+F\left(s^{2} \sigma\left(y_{n}, f u\right)\right) & =\tau+F\left(s^{2} \sigma\left(f x_{n-1}, f u\right)\right) \\
& \leqslant F\left(\max \left\{\sigma\left(g x_{n-1}, g u\right), \sigma\left(g x_{n-1}, f x_{n-1}\right), \sigma(g u, f u), \frac{\sigma\left(g x_{n-1}, f u\right)+\sigma\left(g u, f x_{n-1}\right)}{4 s}\right\}\right) \\
& =F\left(\max \left\{\sigma\left(y_{n-1}, v\right), \sigma\left(y_{n-1}, y_{n}\right), \sigma(v, f u), \frac{\sigma\left(y_{n-1}, f u\right)+\sigma\left(v, y_{n}\right)}{4 s}\right\}\right)
\end{aligned}
$$

for sufficiently large $n \in \mathbb{N}$. Letting $n \rightarrow+\infty$ in the above inequality, we get

$$
\tau+F\left(s^{2} \underline{C}\right) \leqslant F\left(\max \left\{\sigma(v, f u), \frac{\underline{C}}{4 s}\right\}\right) .
$$

If $\max \left\{\sigma(v, f u), \frac{\mathrm{C}}{4 s}\right\}=\sigma(v, f u)$, from $(2.12)$, we get $\tau+F\left(s^{2} \underline{C}\right) \leqslant F(\sigma(v, f u))$, thus $s^{2} \underline{C}<\sigma(v, f u)$, it is a contradiction with $\underline{C} \geqslant \frac{\sigma(v, f u)}{s}$. If $\max \left\{\sigma(v, f u), \frac{\underline{C}}{4 s}\right\}=\frac{\underline{C}}{4 s}$, then we have $\tau+F\left(s^{2} \underline{C}\right) \leqslant F\left(\frac{\mathcal{C}}{4 s}\right)$, which means $s^{2} \underline{C}<\frac{C}{4 s}$, this is a contradiction since $s \geqslant 1$. Therefore, our claim holds, that is, $\sigma(f u, v)=0$, hence, $f u=g u=v$. Thus, $v$ is a point of coincidence of $f$ and $g$. Suppose that the set $C(f, g)$ is g-well-ordered and $v^{\prime}$ is another point of coincidence of $f$ and $g$, then, we can found $u^{\prime} \in X$ such that $f u^{\prime}=g u^{\prime}=v^{\prime}$. Using Lemma 2.4, we get $\sigma\left(v^{\prime}, v^{\prime}\right)=0$. Since $C(f, g)$ is g-well-ordered, we have $g u \asymp g u^{\prime}$. If $\sigma\left(v, v^{\prime}\right)>0$, from (2.1), we obtain

$$
\begin{aligned}
\tau+\mathrm{F}\left(\mathrm{s}^{2} \sigma\left(v, v^{\prime}\right)\right) & =\tau+\mathrm{F}\left(\mathrm{s}^{2} \sigma\left(\mathrm{fu}, \mathrm{fu} \mathbf{u}^{\prime}\right)\right) \\
& \leqslant \mathrm{F}\left(\max \left\{\sigma\left(\mathrm{gu}, \mathrm{gu} \mathbf{u}^{\prime}\right), \sigma(\mathrm{gu}, \mathrm{fu}), \sigma\left(g u^{\prime}, f u^{\prime}\right), \frac{\sigma\left(g u, f u^{\prime}\right)+\sigma\left(g u^{\prime}, \mathrm{fu}\right)}{4 s}\right\}\right) \\
& =\mathrm{F}\left(\max \left\{\sigma\left(v, v^{\prime}\right), \sigma(v, v), \sigma\left(v^{\prime}, v^{\prime}\right), \frac{\sigma\left(v, v^{\prime}\right)+\sigma\left(v^{\prime}, v\right)}{4 s}\right\}\right) \leqslant \mathrm{F}\left(\sigma\left(v, v^{\prime}\right)\right),
\end{aligned}
$$

which yields $s^{2} \sigma\left(v, v^{\prime}\right)<\sigma\left(v, v^{\prime}\right)$. This is a contradiction since $s \geqslant 1$, hence, we have $\sigma\left(v, v^{\prime}\right)=0$, that is, $v=v^{\prime}$. Thus, the point of coincidence of $f$ and $g$ is unique. Moreover, if $f$ and $g$ are weakly compatible, then we have $f v=g v$, let $f v=g v=w$. From the uniqueness of the point of coincidence, we get $f v=g v=w=v$, that is $f v=g v=v$. Therefore, $f$ and $g$ have a unique common fixed point $v$ and $\sigma(v, v)=0$. 
By Theorem 2.5, we can get the following corollary easily.

Corollary 2.6. Let $(\mathrm{X}, \sigma, \preceq)$ be a partially ordered b-metric-like space, $\mathrm{f}$ and $\mathrm{g}$ be self-mappings on $\mathrm{X}$ such that $\mathrm{f}(\mathrm{X}) \subseteq \mathrm{g}(\mathrm{X})$, and $\mathrm{g}(\mathrm{X})$ is complete. If the following conditions hold

(i) $f x \preceq$ fy for $x, y \in X$ such that $g x \preceq g y$;

(ii) there is $\mathrm{x}_{0} \in \mathrm{X}$ such that $\mathrm{gx}_{0} \asymp \mathrm{f \textrm {x } _ { 0 }}$;

(iii) there exist $\tau>0$ and $\mathrm{F} \in \widetilde{\mathbb{F}}$ such that for all $\mathrm{x}, \mathrm{y} \in \mathrm{X}$ with $\mathrm{gx} \asymp \mathrm{gy}$, we have

$$
\sigma(f x, f y)>0 \Rightarrow \tau+F\left(s^{2} \sigma(f x, f y)\right) \leqslant F(\max \{\sigma(g x, g y), \sigma(g x, f x), \sigma(g y, f y)\}) ;
$$

(iv) if $\left\{x_{n}\right\}$ is a sequence in $(X, \sigma)$ which converges to $x \in X$ and $\left\{x_{n}: n \in \mathbb{N}\right\}$ is well-ordered, then $x_{n} \asymp x$ for sufficiently large $\mathrm{n}$,

then $\mathrm{f}$ and $\mathrm{g}$ have a point of coincidence $v \in \mathrm{X}$ and $\sigma(v, v)=0$. Furthermore, if the set of coincidence points of $\mathrm{f}$ and $\mathrm{g}$ is $\mathrm{g}$-well-ordered, then $\mathrm{f}$ and $\mathrm{g}$ have a unique point of coincidence. In addition, if $\mathrm{f}$ and $\mathrm{g}$ are weakly compatible, then $f$ and $g$ have a unique common fixed point.

Taking $\mathrm{g}=\mathrm{I}_{\mathrm{X}}$ in Corollary 2.6, we have the following corollary.

Corollary 2.7. Let $(\mathrm{X}, \sigma, \preceq)$ be a complete partially ordered b-metric-like space, and $\mathrm{f}: \mathrm{X} \rightarrow \mathrm{X}$ be a mapping such that the following conditions hold

(i) $f x \preceq$ fy for $x, y \in X$ such that $x \preceq y$;

(ii) there is $\mathrm{x}_{0} \in \mathrm{X}$ such that $\mathrm{x}_{0} \asymp \mathrm{f \textrm {x } _ { 0 }}$;

(iii) there are $\tau>0$ and $F \in \widetilde{\mathbb{F}}$ such that for all $x, y \in X$ with $x \asymp y$, we have

$$
\sigma(f x, f y)>0 \Rightarrow \tau+F\left(s^{2} \sigma(f x, f y)\right) \leqslant F(\max \{\sigma(x, y), \sigma(x, f x), \sigma(y, f y)\})
$$

(iv) if $\left\{x_{n}\right\}$ is a sequence in $(X, \sigma)$ which converges to $x \in X$ and $\left\{x_{n}: n \in \mathbb{N}\right\}$ is well-ordered, then $x_{n} \asymp x$ for sufficiently large $n$.

Then the mapping $f$ has a point $v \in X$ and $\sigma(v, v)=0$. Furthermore, if the set of fixed points of $f$ is well-ordered, then the mapping $\mathrm{f}$ has a unique fixed point.

Definition 2.8. Let $(X, \sigma, \preceq)$ be a partially ordered b-metric-like space, and $f$ and $g$ be self-mappings on $X$. Suppose that there exist $\tau>0$ and $F \in \widetilde{\mathbb{F}}$ such that

$$
\begin{aligned}
\frac{\sigma(g x, f x)}{2 s}<\sigma(g x, g y) & \Rightarrow \\
\tau+F\left(s^{2} \sigma(f x, f y)\right. & \leqslant F\left(\max \left\{\sigma(g x, g y), \sigma(g x, f x), \sigma(g y, f y), \frac{\sigma(g x, f y)+\sigma(g y, f x)}{4 s}\right\}\right)
\end{aligned}
$$

for all $x, y \in X$ with $g x \asymp g y$. Then the mapping $f$ is called to be a generalized ordered F-g-weak contraction of type (B).

Lemma 2.9. Let $(X, \sigma, \preceq)$ be a partially ordered b-metric-like space, and $f$ and $g$ be self-mappings on $X$ such that $\mathrm{f}$ is a generalized ordered F-g-weak contraction of type (B). If $v \in X$ is a point of coincidence of $f$ and $g$, then $\sigma(v, v)=0$.

Proof. Suppose that $v \in X$ is a point of coincidence of $f$ and $g$, then there is $u \in X$ such that $f u=g u=v$. Suppose $\sigma(v, v)>0$, we have $\frac{\sigma(g u, f u)}{2 s}=\frac{\sigma(g u, g u)}{2 s}<\sigma(g u, g u)$. Due to the fact that $f$ is a generalized ordered F-g-weak contraction of type (B), we have

$$
\begin{aligned}
\tau+F\left(s^{2} \sigma(v, v)\right)=\tau+F\left(s^{2} \sigma(f u, f u)\right. & \leqslant F\left(\max \left\{\sigma(g u, g u), \sigma(g u, f u), \sigma(g u, f u), \frac{\sigma(g u, f u)+\sigma(g u, f u)}{4 s}\right\}\right) \\
& =F\left(\max \left\{\sigma(v, v), \sigma(v, v), \sigma(v, v), \frac{\sigma(v, v)+\sigma(v, v)}{4 s}\right\}\right)=F(\sigma(v, v)),
\end{aligned}
$$

which together with $\mathrm{F}(1)$ yields $s^{2} \sigma(v, v)<\sigma(v, v)$. This is a contradiction since $s \geqslant 1$. Therefore, we get $\sigma(v, v)=0$. 
Theorem 2.10. Let $(X, \sigma, \preceq)$ be a partially ordered b-metric-like space, $f$ and $g$ be self-mappings on $X$ such that $f$ is a generalized ordered F-g-weak contraction of type $(B)$ with $f(X) \subseteq g(X)$, and $g(X)$ is complete. If the following conditions hold

(i) $f x \preceq$ fy for $x, y \in X$ such that $g x \preceq g y$;

(ii) there exists $\mathrm{x}_{0} \in \mathrm{X}$ such that $\mathrm{gx}_{0} \asymp \mathrm{fx}_{0}$;

(iii) if $\left\{x_{n}\right\}$ is a sequence in $(X, \sigma)$ which converges to $x \in X$ and $\left\{x_{n}: n \in \mathbb{N}\right\}$ is well-ordered, then $x_{n} \asymp x$ for sufficiently large $n$,

then $\mathrm{f}$ and $\mathrm{g}$ have a point of coincidence $v \in \mathrm{X}$ and $\sigma(v, v)=0$. Furthermore, if the set of coincidence points of $\mathrm{f}$ and $\mathrm{g}$ is $\mathrm{g}$-well-ordered, then $\mathrm{f}$ and $\mathrm{g}$ have a unique point of coincidence. In addition, if $\mathrm{f}$ and $\mathrm{g}$ are weakly compatible, then $\mathrm{f}$ and $\mathrm{g}$ have a unique common fixed point.

Proof. As in the proof of Theorem 2.5, for $x_{0} \in X$, we can construct a sequence $\left\{y_{n}\right\}$ by $y_{n}=g x_{n}=f x_{n-1}$ and we can get $\left\{y_{n}: n \in \mathbb{N}\right\}$ is well-ordered.

Now, we prove that $f$ and $g$ have a point of coincidence. If $\sigma\left(y_{n}, y_{n+1}\right)=0$ for some $n_{0} \in \mathbb{N}$, hence $\sigma\left(y_{n_{0}}, y_{n_{0}+1}\right)=0$, that is $y_{n_{0}}=y_{n_{0}+1}$, which yields $g x_{n_{0}}=f x_{n_{0}-1}=g x_{n_{0}+1}=f x_{n_{0}}$. Therefore, $x_{n_{0}}$ is a coincidence point of $f$ and $g$, and $g x_{n_{0}}=f x_{n_{0}}$ is a point of coincidence of $f$ and g. Assume $\sigma\left(y_{n}, y_{n+1}\right)>0$ for all $n \in \mathbb{N}$, hence $\sigma\left(g x_{n-1}, f x_{n-1}\right)=\sigma\left(y_{n-1}, y_{n}\right)>0$ for all $n \in \mathbb{N}^{+}$, thus, $\frac{\sigma\left(g x_{n-1}, f x_{n-1}\right)}{2 s}<\sigma\left(g x_{n-1}, f x_{n-1}\right)=\sigma\left(g x_{n-1}, g x_{n}\right)$. Applying (2.14), we obtain

$$
\begin{aligned}
\tau+F\left(s^{2} \sigma\left(y_{n}, y_{n+1}\right)\right) \\
=\tau+F\left(s^{2} \sigma\left(f x_{n-1}, f x_{n}\right)\right) \\
\leqslant F\left(\max \left\{\sigma\left(g x_{n-1}, g x_{n}\right), \sigma\left(g x_{n-1}, f x_{n-1}\right), \sigma\left(g x_{n}, f x_{n}\right), \frac{\sigma\left(g x_{n-1}, f x_{n}\right)+\sigma\left(g x_{n}, f x_{n-1}\right)}{4 s}\right\}\right) \\
=F\left(\max \left\{\sigma\left(y_{n-1}, y_{n}\right), \sigma\left(y_{n-1}, y_{n}\right), \sigma\left(y_{n}, y_{n+1}\right), \frac{\sigma\left(y_{n-1}, y_{n+1}\right)+\sigma\left(y_{n}, y_{n}\right)}{4 s}\right\}\right) \\
\leqslant F\left(\max \left\{\sigma\left(y_{n-1}, y_{n}\right), \sigma\left(y_{n-1}, y_{n}\right), \sigma\left(y_{n}, y_{n+1}\right), \frac{s \sigma\left(y_{n-1}, y_{n}\right)+3 s \sigma\left(y_{n}, y_{n+1}\right)}{4 s}\right\}\right) \\
\leqslant F\left(\operatorname { m a x } \left\{\sigma\left(y_{n-1}, y_{n}\right), \sigma\left(y_{n-1}, y_{n}\right), \sigma\left(y_{n}, y_{n+1}\right),\right.\right. \\
\left.\left.\quad \quad \frac{\operatorname{smax}\left\{\sigma\left(y_{n-1}, y_{n}\right), \sigma\left(y_{n}, y_{n+1}\right)\right\}+3 s \max \left\{\sigma\left(y_{n-1}, y_{n}\right), \sigma\left(y_{n}, y_{n+1}\right)\right\}}{4 s}\right\}\right) .
\end{aligned}
$$

If there exists $n_{0} \in \mathbb{N}$ such that $\max \left\{\sigma\left(y_{n_{0}-1}, y_{n_{0}}\right), \sigma\left(y_{n_{0}}, y_{n_{0}+1}\right)\right\}=\sigma\left(y_{n_{0}}, y_{n_{0}+1}\right)$, by (2.15), we get $\tau+F\left(s^{2} \sigma\left(y_{n_{0}}, y_{n_{0}+1}\right)\right) \leqslant F\left(\sigma\left(y_{n_{0}}, y_{n_{0}+1}\right)\right)$, by $F(1)$, we have $s^{2} \sigma\left(y_{n_{0}}, y_{n_{0}+1}\right)<\sigma\left(y_{n_{0}}, y_{n_{0}+1}\right)$, this is a contradiction. Therefore, we have $\max \left\{\sigma\left(y_{n-1}, y_{n}\right), \sigma\left(y_{n}, y_{n+1}\right)\right\}=\sigma\left(y_{n-1}, y_{n}\right)$ for all $n \in \mathbb{N}^{+}$. From (2.15), we obtain $\tau+F\left(\sigma\left(y_{n}, y_{n+1}\right)\right) \leqslant \tau+F\left(s^{2} \sigma\left(y_{n}, y_{n+1}\right)\right) \leqslant F\left(\sigma\left(y_{n-1}, y_{n}\right)\right)$, so

$$
F\left(\sigma\left(y_{n}, y_{n+1}\right)\right) \leqslant F\left(\sigma\left(y_{n-1}, y_{n}\right)\right)-\tau .
$$

Repeating this process, we get $F\left(\sigma\left(y_{n}, y_{n+1}\right)\right) \leqslant F\left(\sigma\left(y_{0}, y_{1}\right)\right)-n \tau$. The above inequality yields

$$
\lim _{n \rightarrow+\infty} F\left(\sigma\left(y_{n}, y_{n+1}\right)\right)=-\infty
$$

by taking $n \rightarrow+\infty$, thus

$$
\lim _{n \rightarrow+\infty} \sigma\left(y_{n}, y_{n+1}\right)=0 .
$$

Now, we claim $\lim _{m, n \rightarrow+\infty} \sigma\left(y_{m}, y_{n}\right)=0$. Suppose, on the contrary, that there exist $\varepsilon>0$ and sequences $\{p(n)\}$ and $\{q(n)\}$ of natural numbers such that $p(n)>q(n)>n, \sigma\left(y_{p(n)}, y_{q(n)}\right) \geqslant \varepsilon$ and $\sigma\left(y_{p}(n)-1, y_{q}(n)\right)<\varepsilon$ for all $n \in \mathbb{N}$. Since $\lim _{n \rightarrow+\infty} \sigma\left(y_{n}, y_{n+1}\right)=0$, we have $\lim _{n \rightarrow+\infty} \sigma\left(y_{p}(n), y_{p}(n)+1\right)=0$. 


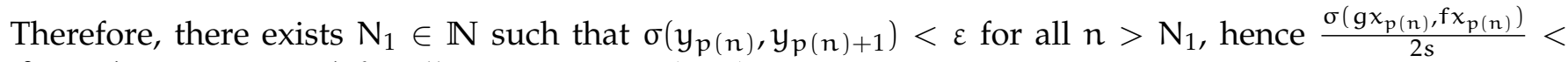
$\frac{\varepsilon}{2 s}<\sigma\left(g x_{p(n)}, g x_{q(n)}\right)$ for all $n>N_{1}$. From (2.14), we get

$$
\begin{aligned}
& \tau+F\left(s^{2} \sigma\left(y_{p}(n)+1, y_{q}(n)+1\right)\right) \\
& =\tau+F\left(s^{2} \sigma\left(f x_{p(n)}, f x_{q(n)}\right)\right) \\
& \leqslant F\left(\operatorname { m a x } \left\{\sigma\left(g x_{p(n)}, g x_{q(n)}\right), \sigma\left(g x_{p(n)}, f x_{p(n)}\right), \sigma\left(g x_{q(n)}, f x_{q(n)}\right)\right.\right. \text {, } \\
& \left.\left.\frac{\sigma\left(g x_{p(n)}, f x_{q(n)}\right)+\sigma\left(g x_{q(n)}, f x_{p(n)}\right)}{4 s}\right\}\right) \\
& =F\left(\operatorname { m a x } \left\{\sigma\left(y_{p(n)}, y_{q(n)}\right), \sigma\left(y_{p}(n), y_{p(n)+1}\right), \sigma\left(y_{q}(n), y_{q}(n)+1\right)\right.\right. \text {, } \\
& \left.\left.\frac{\sigma\left(y_{p(n)}, y_{q(n)+1}\right)+\sigma\left(y_{q}(n), y_{p(n)+1}\right)}{4 s}\right\}\right) \\
& \leqslant F\left(\operatorname { m a x } \left\{\sigma\left(y_{p(n)}, y_{q(n)}\right), \sigma\left(y_{p(n)}, y_{p(n)+1}\right), \sigma\left(y_{q(n)}, y_{q}(n)+1\right),\right.\right. \\
& \left.\left.\frac{s \sigma\left(y_{p(n)}, y_{q(n)}\right)+s \sigma\left(y_{q(n)}, y_{q(n)+1}\right)+s \sigma\left(y_{q(n)}, y_{p(n)}\right)+s \sigma\left(y_{p(n)}, y_{p(n)+1}\right)}{4 s}\right\}\right) \text {. }
\end{aligned}
$$

From the triangle inequality, we get

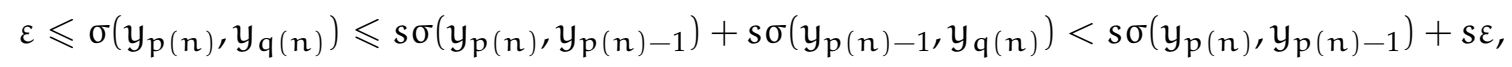

and

$$
\sigma\left(y_{p(n)+1}, y_{q(n)+1}\right) \leqslant s \sigma\left(y_{p(n)+1}, y_{p(n)}\right)+s^{2} \sigma\left(y_{p(n)}, y_{q(n)}\right)+s^{2} \sigma\left(y_{q}(n), y_{q}(n)+1\right),
$$

these inequalities together with (2.17) show that sequences $\left\{\sigma\left(y_{p}(n), y_{q}(n)\right)\right\}$ and $\left\{\sigma\left(y_{p}(n)+1, y_{q}(n)+1\right)\right\}$ are bounded, thus, they have inferior limits, respectively, and we let

$$
\liminf _{n \rightarrow+\infty} \sigma\left(y_{p}(n), y_{q}(n)\right)=\underline{a} ; \quad \liminf _{n \rightarrow+\infty} \sigma\left(y_{p}(n)+1, y_{q}(n)+1\right)=\underline{b} .
$$

Letting $n \rightarrow+\infty$ in (2.18), by (2.19), we obtain $\tau+F\left(s^{2} \underline{b}\right) \leqslant F(\underline{a})$, which means $s^{2} \underline{b}<\underline{a}$. By the triangle inequality, we get

$$
\sigma\left(y_{p(n)}, y_{q(n)}\right) \leqslant s \sigma\left(y_{p(n)}, y_{p(n)+1}\right)+s^{2} \sigma\left(y_{p(n)+1}, y_{q(n)+1}\right)+s^{2} \sigma\left(y_{q(n)+1}, y_{q(n)}\right),
$$

letting $n \rightarrow+\infty$ in the above inequality, we have $\underline{a} \leqslant s^{2} \underline{b}$, which is a contradiction with $s^{2} \underline{b}<\underline{a}$, therefore $\lim _{m, n \rightarrow+\infty} \sigma\left(y_{m}, y_{n}\right)=0$, hence, the sequence $\left\{y_{n}\right\}=\left\{g x_{n}\right\}$ is a Cauchy sequence in $g(X)$. By completeness of $g(X)$, there exist $u, v \in X$ such that $v=g u$ and

$$
\lim _{n \rightarrow+\infty} \sigma\left(y_{n}, v\right)=\lim _{n \rightarrow+\infty} \sigma\left(g x_{n}, g u\right)=\lim _{m, n \rightarrow+\infty} \sigma\left(y_{n}, y_{m}\right)=\sigma(v, v)=0 .
$$

Now, we prove that, for every $n \in \mathbb{N}$,

$$
\frac{\sigma\left(g x_{n}, f x_{n}\right)}{2 s}<\sigma\left(g x_{n}, g u\right) \text { or } \frac{\sigma\left(f x_{n}, f x_{n+1}\right)}{2 s}<\sigma\left(f x_{n}, g u\right) .
$$

For that, we argue it by contradiction. We assume that there exists $m \in \mathbb{N}$ such that

$$
\frac{\sigma\left(g x_{m}, f x_{m}\right)}{2 s} \geqslant \sigma\left(g x_{m}, g u\right) \text { and } \frac{\sigma\left(f x_{m}, f x_{m+1}\right)}{2 s} \geqslant \sigma\left(f x_{m}, g u\right) .
$$

Applying (2.16), we get $F\left(\sigma\left(y_{m+1}, y_{m+2}\right)\right)<F\left(\sigma\left(y_{m}, y_{m+1}\right)\right)$, hence, $\sigma\left(y_{m+1}, y_{m+2}\right)<\sigma\left(y_{m}, y_{m+1}\right)$, that is,

$$
\sigma\left(f x_{m}, f x_{m+1}\right)<\sigma\left(g x_{m}, f x_{m}\right) .
$$


Combining (2.22) and (2.23), we obtain

$$
\begin{aligned}
\sigma\left(g x_{m}, f x_{m}\right) \leqslant s \sigma\left(g x_{m}, g u\right)+s \sigma\left(g u, f x_{m}\right) & \leqslant \frac{1}{2} \sigma\left(g x_{m}, f x_{m}\right)+\frac{1}{2} \sigma\left(f x_{m}, f x_{m+1}\right) \\
& <\frac{1}{2} \sigma\left(g x_{m}, f x_{m}\right)+\frac{1}{2} \sigma\left(g x_{m}, f x_{m}\right)=\sigma\left(g x_{m}, f x_{m}\right),
\end{aligned}
$$

which is a contradiction. Hence (2.21) holds. Now, we prove $\sigma(g u, f u)=0$. Arguing by contradiction, we assume $\sigma(\mathrm{gu}, \mathrm{fu})>0$. Next, we discuss the following cases.

Case 1. Suppose that the LHS of (2.21) holds, that is, $\frac{\sigma\left(g x_{n}, f x_{n}\right)}{2 s}<\sigma\left(g x_{n}, g u\right)$ for $n \in \mathbb{N}$, then by (2.14), we have

$$
\begin{aligned}
\tau+F\left(s^{2} \sigma\left(f x_{n}, f u\right)\right) \leqslant & F\left(\max \left\{\sigma\left(g x_{n}, g u\right), \sigma\left(g x_{n}, f x_{n}\right), \sigma(g u, f u), \frac{\sigma\left(g x_{n}, f u\right)+\sigma\left(g u, f x_{n}\right)}{4 s}\right\}\right) \\
\leqslant & F\left(\operatorname { m a x } \left\{\sigma\left(g x_{n}, g u\right), \sigma\left(g x_{n}, f x_{n}\right), \sigma(g u, f u),\right.\right. \\
& \left.\left.\frac{s \sigma\left(g x_{n}, g u\right)+s \sigma(g u, f u)+s \sigma\left(g u, g x_{n}\right)+s \sigma\left(g x_{n}, f x_{n}\right)}{4 s}\right\}\right)
\end{aligned}
$$

for sufficiently large $n$. From (2.17) and (2.20), there exists $N_{2} \in \mathbb{N}$ such that $\sigma\left(g x_{n}, g u\right)<\sigma(g u, f u)$ and $\sigma\left(g x_{n}, f x_{n}\right)<\sigma(g u, f u)$. By (2.24), we have

$$
\tau+F\left(s^{2} \sigma\left(f x_{n}, f u\right)\right)<F(\sigma(g u, f u))
$$

for all $n>N_{2}$. Since $\sigma\left(f x_{n}, f u\right) \leqslant s \sigma\left(f x_{n}, g x_{n}\right)+s^{2} \sigma\left(g x_{n}, g u\right)+s^{2} \sigma(g u, f u)$, then, the sequence $\left\{\sigma\left(f x_{n}, f u\right)\right\}$ is bounded, hence we can let $\liminf _{n \rightarrow+\infty} \sigma\left(f x_{n}, f \mathfrak{u}\right)=\underline{c}$. Taking $n \rightarrow+\infty$ in $(2.25)$, we have $\tau+F\left(s^{2} \underline{c}\right)<$ $\mathrm{F}(\sigma(g u, f u))$, hence $s^{2} \underline{c}<\sigma(g u, f u)$. On the other hand, since $\sigma(g u, f u) \leqslant s \sigma\left(g u, g x_{n}\right)+s^{2} \sigma\left(g x_{n}, f x_{n}\right)+$ $s^{2} \sigma\left(f x_{n}, f u\right)$, letting $n \rightarrow+\infty$, we get $\sigma(g u, f u) \leqslant s^{2} \underline{c}$, which is a contradiction with $s^{2} \underline{c}<\sigma(g u, f u)$.

Case 2. Suppose that the RHS of (2.21) is satisfied, that is, $\frac{\sigma\left(f x_{n}, f x_{n+1}\right)}{2 s}<\sigma\left(g x_{n+1}, g u\right)$, hence

$$
\frac{\sigma\left(g x_{n+1}, f x_{n+1}\right)}{2 s}<\sigma\left(g x_{n+1}, g u\right)
$$

for $n \in \mathbb{N}$, then we have

$$
\begin{aligned}
\tau+F\left(s^{2} \sigma\left(f x_{n+1}, f u\right)\right) \leqslant & F\left(\operatorname { m a x } \left\{\sigma\left(g x_{n+1}, g u\right), \sigma\left(g x_{n+1}, f x_{n+1}\right), \sigma(g u, f u),\right.\right. \\
& \left.\left.\frac{\sigma\left(g x_{n+1}, f u\right)+\sigma\left(g u, f x_{n+1}\right)}{4 s}\right\}\right) \\
\leqslant & \left.F\left({\max \left\{\sigma\left(y_{n+1}, g u\right), \sigma\left(y_{n+1}, y_{n+2}\right), \sigma(g u, f u),\right.}_{4 s}\left(g u, y_{n+2}\right)\right\}\right) \\
& \frac{\sigma\left(y_{n+1}, f u\right)+\sigma\left(y_{n+1}, y_{n+2}\right), \sigma(g u, f u),}{4 s}
\end{aligned}
$$

for sufficiently large $n$.

Since $\sigma\left(y_{n}, f u\right) \leqslant s \sigma\left(y_{n}, g u\right)+s \sigma(g u, f u)$, then the sequence $\left\{\sigma\left(y_{n}, f u\right)\right\}$ is bounded by (2.20). Let $\liminf _{n \rightarrow+\infty} \sigma\left(y_{n}, f u\right)=\underline{d}$. Taking $n \rightarrow+\infty$ in $(2.26)$, we get $\tau+F\left(s^{2} \underline{d}\right) \leqslant F(\sigma(g u, f u))$, it yields $s^{2} \underline{d}<\sigma(g u, f u)$. On the other hand, since $\sigma(g u, f u) \leqslant s \sigma\left(g u, y_{n}\right)+s \sigma\left(y_{n}, f u\right)$, by taking $n \rightarrow+\infty$, we obtain $\sigma(g u, f u) \leqslant$ $s \underline{d}$, which is contradiction with $s^{2} \underline{d}<\sigma(g u, f u)$. From Cases 1 and 2 , we get $\sigma(g u, f u)=0$, thus $g u=$ $f u=v$, i.e., $v$ is a point of coincidence of $f$ and $g$. Suppose that $\mathrm{C}(f, g)$ is g-well-ordered and $v^{\prime}$ is another point of coincidence of $f$ and $g$, then there exists $u^{\prime}$ such that $f u^{\prime}=g u^{\prime}=v^{\prime}$. Using Lemma 2.9, we 
get $\sigma\left(v^{\prime}, v^{\prime}\right)=0$. Since $\mathrm{C}(\mathrm{f}, \mathrm{g})$ is g-well-ordered, we get $g u \asymp g u^{\prime}$. If $\sigma\left(v, v^{\prime}\right)>0$, since $\frac{\sigma\left(g u, f u^{\prime}\right)}{2 s}<$ $\sigma\left(g u, f u^{\prime}\right)=\sigma\left(g u, g u^{\prime}\right)$, from (2.14), we obtain

$$
\begin{aligned}
\tau+\mathrm{F}\left(\mathrm{s}^{2} \sigma\left(v, v^{\prime}\right)\right) & =\tau+\mathrm{F}\left(\mathrm{s}^{2} \sigma\left(\mathrm{fu}, \mathrm{fu} \mathbf{u}^{\prime}\right)\right) \\
& \leqslant \mathrm{F}\left(\max \left\{\sigma\left(\mathrm{gu}, \mathrm{gu} \mathbf{u}^{\prime}\right), \sigma(\mathrm{gu}, \mathrm{fu}), \sigma\left(g u^{\prime}, \mathrm{fu} \mathbf{u}^{\prime}\right), \frac{\sigma\left(g u, f u^{\prime}\right)+\sigma\left(g u^{\prime}, \mathrm{fu}\right)}{4 s}\right\}\right) \\
& =\mathrm{F}\left(\max \left\{\sigma\left(v, v^{\prime}\right), \sigma(v, v), \sigma\left(v^{\prime}, v^{\prime}\right), \frac{\sigma\left(v, v^{\prime}\right)+\sigma\left(v^{\prime}, v\right)}{4 s}\right\}\right) \leqslant \mathrm{F}\left(\sigma\left(v, v^{\prime}\right)\right)
\end{aligned}
$$

which yields $s^{2} \sigma\left(v, v^{\prime}\right)<\sigma\left(v, v^{\prime}\right)$. This is a contradiction since $s \geqslant 1$, hence, we have $\sigma\left(v, v^{\prime}\right)=0$, that is $v=v^{\prime}$. Thus, the point of coincidence of $f$ and $g$ is unique. Furthermore, if $f$ and $g$ are weakly compatible, then we have $f v=g v$, let $f v=g v=w$. By the uniqueness of the point of coincidence of $f$ and $g$, we get $f v=g v=w=v$, that is $f v=g v=v$. Therefore, $f$ and $g$ have a unique common fixed point $v$ and $\sigma(v, v)=0$.

Now, we use an example to illustrate the validity of our main result.

Example 2.11. Let $X=\{0,1,5,10\}$. Define $\sigma: X \times X \rightarrow \mathbb{R}^{+}$by $\sigma(x, y)=(\max \{x, y\})^{2}$. Then $(X, \sigma)$ is a complete b-metric-like space with the constant $s=2$. Define a partial order relation " $\preceq$ " on $X$ by $\preceq=\{(0,0),(1,1),(5,5),(10,10),(0,5),(5,1),(0,1)\}$. We define $f: X \rightarrow X$ as follows: $f 0=0, f 1=0, f 5=$ $0, f 10=1$. The function $F$ is defined by $F(\alpha)=\ln \alpha$. Since $\sigma(f 0, f 0)=\sigma(f 1, f 1)=\sigma(f 5, f 5)=\sigma(f 0, f 5)=$ $\sigma(f 5, f 1)=\sigma(f 0, f 1)=0, \sigma(f 10, f 10)=1$, and $F(\max \{\sigma(10,10), \sigma(10, f 10), \sigma(10, f 10)\})-F(4 \sigma(f 10, f 10))=$ $\mathrm{F} 100-\mathrm{F} 4=\ln 25$, then, the condition of (2.13) is satisfied for $\tau \in(0, \ln 25)$.

At the same time, we can see that all other conditions of Corollary 2.7 are satisfied and 0 is the unique fixed point of $f$.

\section{Acknowledgment}

The authors thank the referees for their valuable comments and suggestions to improve this paper. The research was supported by the National Natural Science Foundation of China $(11361042,11661053)$ and supported partly by the Provincial Natural Science Foundation of Jiangxi, China (20142BAB201007).

\section{References}

[1] M. Abbas, G. Jungck, Common fixed point results for noncommuting mappings without continuity in cone metric spaces, J. Math. Anal. Appl., 341 (2008), 416-420. 2.3

[2] M. A. Alghamdi, N. Hussain, P. Salimi, Fixed point and coupled fixed point theorems on b-metric-like spaces, J. Inequal. Appl., 2013 (2013), 25 pages. 1, 1.1, 1.2, 1

[3] H. H. Alsulami, E. Karapınar, H. Piri, Fixed points of generalized F-Suzuki type contraction in complete b-metric spaces, Discrete Dyn. Nat. Soc., 2015 (2015), 8 pages. 1

[4] H. H. Alsulami, E. Karapınar, H. Piri, Fixed points of modified F-contractive mappings in complete metric-like spaces, J. Funct. Spaces, 2015 (2015), 9 pages.

[5] M. Cosentino, P. Vetro, Fixed point results for F-contractive mappings of Hardy-Rogers-type, Filomat, 28 (2014), 715-722. 1

[6] S. Czerwik, Contraction mappings in b-metric spaces, Acta Math. Inform. Univ. Ostraviensis, 1 (1993), 5-11. 1

[7] D. Gopal, M. Abbas, D. K. Patel, C. Vetro, Fixed points of $\alpha$-type F-contractive mappings with an application to nonlinear fractional differential equation, Acta Math. Sci. Ser. B Engl. Ed., 36 (2016), 957-970. 1

[8] S. K. Malhotra, S. Radenović, S. Shukla, Some fixed point results without monotone property in partially ordered metriclike spaces, J. Egyptian Math. Soc., 22 (2014), 83-89. 1, 1, 1.5, 1, 1.6

[9] S. G. Matthews, Partial metric topology, Papers on general topology and applications, Flushing, NY, (1992), Ann. New York Acad. Sci., New York Acad. Sci., New York, 728 (1994), 183-197. 1

[10] H. Piri, P. Kumam, Some fixed point theorems concerning F-contraction in complete metric spaces, Fixed Point Theory Appl., 2014 (2014), 11 pages. 1

[11] H. Piri, P. Kumam, Wardowski type fixed point theorems in complete metric spaces, Fixed Point Theory Appl., 2016 (2016), 12 pages. 1 
[12] D. Wardowski, Fixed points of a new type of contractive mappings in complete metric spaces, Fixed Point Theory Appl., 2012 (2012), 6 pages. $1,1.3$

[13] D. Wardowski, N. Van Dung, Fixed points of F-weak contractions on complete metric spaces, Demonstr. Math., 1 (2014), 146-155. 1, 1.4 